\title{
LA SITUACIÓN DE LAS ANNONACEAE EN MÉXICO: PRINCIPALES PLAGAS, ENFERMEDADES Y SU CONTROL
}

\author{
LIBRADO VIDAL HERNÁNDEZ², HÉCTOR LÓPEZ MOCTEZUMA² \\ NAYELLI AYATZOL VIDAL MARTÍNEZ², ROMEO RUIZ BELLO², \\ DORIS GUADALUPE CASTILLO ROCHA ${ }^{2}$, ROBERTO GREGORIO CHIQUITO CONTRERAS ${ }^{2}$
}

RESUMEN -La importancia económica de las especies de la familia Annonaceae en México es diversa y no se restringe a las especies comestibles, pues además incluye especies con propiedades aromáticas para la extracción de aceites esenciales, medicinales, insecticidas y tóxicos a peces. En general esta familia no se ha formalizado agronómica ni económicamente, sin embargo, presenta grandes perspectivas dentro de programas de mejoramiento genético, en el uso como portainjertos o bien como cultivos alternativos. Las principales plagas asociadas al género Annona son: Bephratelloides cubensis Ashmead, Cerconota anonella Sepp., Corythuca gossypii Fab., Planococcus citri, Chrysobotris sp., Talponia batesi Heinrich., Acantocephala femorata Fab.. Las principales enfermedades de las anonáceas reportadas son: Colletotrichum gloeosporioides Penz, Rhizopus stolonifer Ehr., Phyllosticta sp., Pestalotia sp., Macrophoma sp., Fusarium sp y Phytopthora sp.. Siendo la primera la principal enfermedad de mayor importancia en el cultivo del guanábano dado que disminuye el rendimiento y calidad de los frutos. En chirimoyo y guanábano es muy poca la información bibliográfica existente sobre plagas y enfermedades, y en las demás especies de Annona es nula. No se han realizado evaluaciones de las pérdidas que ocasionan las plagas y enfermedades en las Anonáceas, ocasionando un desconocimiento pleno sobre los daños ocasionados por este factor biótico.

Termos para indexacion: Anonáceas, especies, enfermedades, cultivo.

\section{A SITUAÇÃO DAS ANNONACEAE NO MÉXICO: PRINCIPAIS PRAGAS, DOENÇAS E CONTROLE}

\begin{abstract}
RESUMO-A importância econômica das espécies da família Annonaceae no México é diversificada e inclui espécies com propriedades: aromáticas para a extração de óleos essenciais, medicinais, inseticidas e tóxicas para os peixes. Em geral, esta família não tem sido formalizada quer agronômica ou economicamente, no entanto, tem grandes perspectivas em programas de melhoramento genético, em uso como porta-enxertos ou como cultura alternativa. As principais pragas que estão associadas com o gênero Annona são: Bephratelloides cubensis Ashmead, Cerconota anonella Sepp, Corythuca gossypii Fab., Planococcus citri, Chrysobotris sp., Talponia batesi Heinrich., Acantocephala femorata Fab.. Os principais fungos reportados são: Colletotrichum gloeosporioides Penz, Rhizopus stolonifer Ehr, Phyllosticta sp., Pestalotia sp., Macrophoma sp., Fusarium sp. e Phytophthora sp.. O primeiro é o principal fungo e tem grande importância no cultivo de graviola, uma vez que diminui o rendimento e a qualidade dos frutos. Em cherimóia e graviola existe pouca informação documental sobre pragas e doenças, e em outras espécies de Annona são nulas. Não houve avaliação dos prejuízos causados pragas e doenças nas anonáceas, resultando em completo desconhecimento sobre os danos causados pelo fator biótico.
\end{abstract}

Termos para indexação: Annonaceae, espécies, doenças, cultivo.

\footnotetext{
${ }^{1}$ Palestra Anonáceas - V Congresso Internacional \& Encontro Brasileiro sobre Annonaceae: do gene à exportação (19 a 23 de Agosto de 2013). Botucatu-SP.

${ }^{2}$ Académicos de la Facultad de Ciencias Agrícolas. Universidad Veracruzana Campus Xalapa.Xalapa Veracruz, México. E-mails: lvidal152@hotmail.com; hlopez@uv.mx; angelitonv@gmail.com; rorube@hotmail.com; doriscr@gmail.com; contrerasch@yahoo.com
} 


\section{INTRODUCCIÓN}

En México se producen actualmente más de treinta especies de frutales, muchas de estas adaptadas a clima cálido y semicálido. La familia Annonaceae, se caracteriza por crecer principalmente en los trópicos, comprende 130 géneros y aproximadamente 2,300 especies (GONZÁLEZ et al., 1999), dentro de esta familia, los cuatros géneros de mayor importancia económica, por su calidad frutícola y potencial farmacéutico, son Annona, Rollinea, Uvaria y Asimina. El género Annona comprende alrededor de 120 especies de clima tropical y subtropical, principalmente de América. De acuerdo al Sistema Integrado de Información Taxonómica (SSIT-CONABIO), actualmente se han aceptado para México 13 géneros y 37 especies de la familia Annonaceae. De acuerdo con la base de datos de la Red Mundial de Información sobre Biodiversidad (REMIB), de las colecciones de los herbarios se definieron 14 géneros y 62 especies distribuidas en varios estados de la República Mexicana (ANDRÉS y ANDRÉS, 2011). La distribución de las especies en México se encuentra bien definida, así tenemos que el chirimoyo (Annona cherimola Mill.) es la única especies de las anonáceas que prospera en altitudes de 1400 a $1800 \mathrm{~m}$. se desarrolla en el estado de Michoacán, Estado de México, Veracruz y Morelos. El papause (Annona diversifolia Saff) se distribuye principalmente en los estados de Chiapas, Oaxaca y Guerrero, la chirimuya (Annona escleroderma) en Chiapas. El saramuyo (Annona squamosa L.) en Quintana Roo, Yucatán y Campeche. El anono de corcho (Annona glabra L.) y chirimoyito (Annona globiflora) en Veracruz. El anono (Annona reticulata L.), chincuya (Annona purpurea Moc \& Sessé) y el guanábano (Annona muricata L.) son especies cosmopolitas, debido a que presentan un rango más amplio de adaptabilidad edáfica, así lo podemos encontrar en todas las entidades antes mencionadas. Las especies que han alcanzado mayor desarrollo comercial son guanábano, chirimoyo y atemoya, y de mercado local el saramuyo y papause (VIDAL, 2006).

Las anonas como el chirimoyo (Annona cherimola Mill.), y el guanábano (Annona muricata L.) se cultivan en áreas pequeñas y dispersas. Estas se comercializan en mercado nacional e internacional. Las siguientes especies son propiamente de recolección y únicamente se distribuyen de manera local como es el caso del papause (Annona diversifolia Saff) y el saramuyo (Annona squamosa L.) se desarrollan a nivel de huertos familiares, en cambio el anono (Annona reticulata L.), la ilama (Annona purpurea Moc and Sessé) y anono de corcho (Annono glabra L.) se distribuyen de forma aislada o intercalado con otras especies frutales o bien en linderos o cercas vivas. La chirimuya (Annona escleroderma) proporciona sombra al café y cacao, y fue introducida por el autor en el 2008, del Ejido Francisco Sarabia municipio de Tuzantán, Chiapas (región de Huixtla) en tanto que el chirimoyito (Annona globiflora) se desarrolla de manera silvestres formando parte de terrenos destinados a la ganadería en la zona centro del estado de Veracruz. El guanábano cimarrón (Annona montana) fue introducido por el autor de Cuba en 1979. El chirimoyo de tierra caliente (Annona lutescens) el autor la introdujo de Tilapa, Puebla en 2009.

El cultivo de estas especies de Anonáceas presenta grandes perspectivas en México, por una parte para emplearse en los programas de mejoramiento genético, en el uso como portainjertos o bien como cultivos alternativos ya que poseen un excelente aroma y sabor al paladar. Sin embargo, la importancia económica de las especies de la familia Annonaceae es diversa y no se restringe a las especies comestibles, pues además incluye especies con propiedades aromáticas para la extracción de aceites esenciales, propiedades medicinales, propiedades tóxicas a peces y propiedades insecticidas. A pesar de las importantes características anteriormente mencionadas, en general el cultivo de las Annonaceae no se ha formalizado agronómica y económicamente en México.

\section{SITUACIÓN ACTUAL DE LAS ANONÁCEAS EN MÉXICO}

El primer reporte del guanábano en México se presentó en el IV Simposium Nacional de Parasitología Agrícola, celebrado en la ciudad de Veracruz y en donde se señala los problemas fitosanitarios de la guanábana (PONCE, 1976). Durante el periodo 1977-1978, se realizaron una serie de experimentos encaminados con el control del barrenador del fruto: evaluación de insecticidas $y$ embolsados de frutos como medidas de control de esta plaga. De forma paralela, se trabajó con programa de selección de criollos resistentes a plagas y enfermedades (PONCE y VIDAL, 1981). Con la finalidad de controlar el barrenador de la semilla, se estableció un experimento sobre el Efecto de los Reguladores de Crecimiento en la Formación de Frutos Partenocárpicos en Guanábana (Annona muricata L.) (VIDAL, 1981). En 1983, se da a conocer un folleto sobre "El cultivo de la guanábana 
en México, y menciona el proceso productivo de este frutal (VIDAL, 1983). La mayor parte de las investigaciones realizadas ha sido encaminada principalmente a problemas de plagas y a la selección de fenotipos sobresalientes en guanábano.

Dos escritos que por primera vez se habla del género Annona: "Importancia y Distribución de las Anonáceas en México"y “La Reproducción sexual y Multiplicación Vegetativa de las Anonáceas" ambos se dieron a conocer en el VII Curso de Actualización Frutícola "La Fruticultura y sus Perspectivas para el siglo XXI" Fundación Salvador Sánchez Colín. CICTAMEX, S. C. Coatepec Harinas, México (VIDAL, 1994).

Durante el periodo de 1995-1996 surge un grupo de investigadores interesados en el estudio de las anonáceas, mismo que se le denominó “Grupo de Trabajo de Anonáceas" con sede en la Universidad Autónoma Chapingo. Este mismo grupo organizó el Primer Congreso Internacional de Anonáceas en 1997 con sede en la UACH. Se dan a conocer 42 trabajos de investigación sobre las especies del género Annona. Actualmente se han celebrado cuatro Congresos Internacionales y seis Nacionales. La mayoría de las investigaciones presentadas en estos Congresos de las anonáceas ha sido encaminada hacia el diagnóstico, problemática, caracterización de las especies, distribución, importancia económica, epidemiología, etología de los insectos, etc. Todo esto, es la base fundamental para establecer estrategia de manejo necesaria e implementar mecanismo para la solución de una problemática fitosanitaria real y compleja dentro de las anonáceas. Poco se ha investigado sobre el manejo de plagas y enfermedades para reducir su incidencia. No se tiene información sobre la estimación de daños, el problema de la incidencia y severidad, todos ellos son temas que deberá abordase de manera inmediata, dado que el cultivo tiende a expandirse, pero con escasas experiencias en lo referente al manejo del cultivo.

Actualmente, existen plantaciones comerciales de chirimoya en Michoacán y Estado de México y de guanábano en los estados de Nayarit, Colima, Veracruz, Tabasco, Oaxaca, Campeche, Guerrero y Chiapas. En México se cultiva 7,250 ha de estos frutales, con un volumen estimado de 54,375 toneladas. El estado de Nayarit actualmente es el principal productor de guanábana, con una superficie de 2300 ha., y un volumen de producción de 16,100 toneladas anuales. Los estados de Nayarit, Colima y Veracruz cubren el $85 \%$ de la producción nacional. Por otro lado, Veracruz ocupa el tercer lugar en superficie sembrada de este frutal, y se encuentran 11especies de anonas en su territorio: guanábana (A. muricata), chirimoya (A. cherimola), saramuyo (A. squamosa), papause (A. diversifolia), anona $(A$. reticulata), chincuya ( $A$. purpurea), chirimoyito (A. globiflora), chirimoya de tierra caliente $(A$. lutescens), chirimuya (A. escleroderma), guanábana cimarrona (A. montana), anona de corcho (A. glabra).

\section{Problemática fitosanitaria de las anonáceas en México}

Un factor que constituye una limitante severa en la producción de frutales en América Central y en el resto del mundo son las plagas y enfermedades. La siembra intensiva (monocultivo) y la poca tecnología de algunos frutales, unido al desequilibrio ecológico producido por prácticas inadecuadas de manejo de insectos plagas y enfermedades, han ocasionado que algunas plagas secundarias se conviertan en primarias para muchos de estos cultivos (COTO y SAUNDERS, 2001).

Las pérdidas ocasionadas por plagas y enfermedades a la producción de todos los cultivos en el mundo se estiman en un 35\% (PIMENTEL, 1995); las plagas insectiles ocasionan un $13 \%$ de pérdidas, las enfermedades un $12 \%$ y las malezas un $10 \%$ (CRAMER, 1967, citado por OROZCO et al., 1998). Anualmente, se aplican 2.5 millones de toneladas de plaguicidas a escala mundial. Del total de plaguicidas aplicados, un 59 a $60 \%$ son herbicidas, 20 a $30 \%$ insecticidas y 10 a $20 \%$ fungicidas (OROZCO et al., 1998).

La mayor cantidad de plaguicidas se aplica en cultivos de alto valor económico (algodón, maíz, arroz, frutales y hortalizas) y sólo una cantidad insignificante del producto químico (menos del $0.1 \%$ ) logra llegar a la plaga objetivo (PIMENTEL y LEVITAN, 1986). Esto significa que más del $99.9 \%$ del plaguicida aplicado se pierde en el medio ambiente, donde puede causar efectos adversos a la fauna benéfica, contaminación del suelo, agua y atmósfera (PIMENTEL et al., 1993).

Se consideran al menos 296 especies de artrópodos asociados con el género Annona en los países del trópico. La familia de insectos frecuentemente observados son Coccidae, Noctuidae, Oecophoridae y Eurytomidae.

En México, el impacto de las plagas barrenadores de anonáceas: Talponia batesi presente en chirimoyo y en guanábano, Bephratelloides cubensis (Fig 1) y Cerconota anonella) (Fig. 2) ha orillado a productores a usar diferentes estrategias para su manejo y/o control como la aplicación 
de plaguicidas, mismos que han demostrado ser ineficientes al propiciar la aparición de insectos resistentes e intensificar la incidencia y al contaminar los suelos (PEÑA y NAGEL, 1988; BRECHELT, 2004).

En diversos cultivos se han realizado hasta 6 aplicaciones por año sin lograr un control efectivo (HERNÁNDEZ et al., 2008). No obstante, diversos productores en el país siguen utilizando plaguicidas con la esperanza de disminuir el impacto de las plagas a sus cultivos, desconociendo si en realidad son eficientes, y causando un daño a la naturaleza. Por ello, la búsqueda de alternativas menos dañinas en el control de plagas es una estrategia viable. Las investigaciones enfocadas a la búsqueda de metodologías eficientes para el control de insectos barrenadores de anonáceas constituyen una prioridad en países como Costa Rica, Venezuela, Colombia, Trinidad y Tobago, USA, México, Puerto Rico y Brasil.

Es indiscutible la importancia económica que representan las tres plagas para el cultivo de las anonáceas, considerando las características mismas de las plagas, el control químico es un método no muy recomendado. Aparentemente no existe hasta la fecha un tratamiento para el control efectivo del complejo barrenador de anonáceas, lo cual hace el problema mayor debido a que las zonas afectadas se convierten en foco de infección para las futuras plantaciones de tipo comercial (ALOMÍA, 1980; BOSCAN y GODOY, 1989).

Actualmente en México no hay un estudio detallado sobre la entomofauna asociada con las especies de Annona. Bautista et al., (2003), reporta seis plagas en el cultivo del guanábano en Nayarit y 4 insectos de baja importancia económica.

Un estudio sobre la entomofauna asociada con las especies de Annona, distribuidas en el estado de Veracruz fue realizado por Illescas (2009).

\section{Bephratelloides cubensis Ashmead}

(Himenóptera:Eurytomidae). Hospederos: $A$. muricata, A. reticulata, A. diversifolia, A. squamosa, A. cherimola, A. glabra, A. globiflora. Distribución altitudinal (msnm): 3-980.

Cerconota anonella Sepp. (Lepidóptera: Oecophoridae): Hospederos: A. muricata, $A$. reticulata, $A$. diversifolia, $A$. squamosa, $A$. cherimola, A. glabra, A. globiflora y A. purpurea: Distribución altitudinal (msnm): 3-1460.

Talponia batesi Heinrich.

(Lepidóptera:Tortricidae): Hospederos: $A$. cherimola. Distribución altitudinal (msnm):14601736.
Acantocephala femorata Fab. (Hemíptera: Coreidae): Hospederos: A. muricata. Distribución altitudinal (msnm): 170-173.

Pseudococcus citri Rossi. (Hemíptera: Pseudococcidae): Hospederos: A. muricata y A. squamosa: Distribución altitudinal (msnm): 62700.

Thecla ortygnus Cramer (=Oenomaus ortygnus). (Lepidóptera:Lycaenidae): Hospederos: A. glabra, A. globiflora y A. muricata. Distribución altitudinal (msnm): 3-980.

Membracis mexicana Guerin.

(Hemíptera:Membracidae). Hospedero: A. muricata. Distribución altitudinal (msnm): 102-920.

Saissetia sp. (Hemíptera: Coccidae). Hospederos: A. muricata y A. reticulata. Distribución altitudinal (msnm): 102-700.

Optatus palmaris Pascoe. (Coleóptera: Curculionidae). Hospedero: A. squamosa. Distribución altitudinal (msnm): 170.

Lonchaeidae: Díptera*. Hospederos: $A$. muricata, A. squamosa, A. reticulata, A. purpurea, $A$. diversifolia, A. cherimola, A. globiflora y A. glabra. Distribución altitudinal (msnm): 3-1736.

\section{* Reportado por primera vez en México.}

Pyralidae: Lepidóptera*. Hospederos: $A$. muricata, A. squamosa, A. purpurea y A. reticulata. Distribución altitudinal (msnm): 62-1160.

* Reportado por primera vez en México.

Dentro de esta diversidad de insectos dañinos los que más se destacan por producir daños económicos al cultivo son la avispita o barrenador de la semilla del fruto del guanábano Bephratelloides cubensis, el perforador del fruto o palomilla Cerconota anonella y la chinche de encaje Corythuca gossypii. Asimismo, Vidal (2006), reporta pérdidas del $40 \%$ por presencia de plagas y $50 \%$ por incidencia de enfermedades.

En un monitoreo realizado en diciembre de 2012 en una plantación comercial de guanábano ubicado en la localidad de Blanca Espuma, municipio de Alto Lucero, con manejo de cultivo, se encontró una alta incidencia del barrenador de la semilla $(70 \%)$ y baja incidencia del perforador del fruto 
$(10 \%)$ (Cuadro 1).

Como se señaló anteriormente, en una plantación con manejo de cultivo, destaca la alta incidencia de la avispita en toda la escala de daños. Pero lo más sobresaliente es que la escala 3, 4 y 5 presentaron numerosos orificios en la superficie del fruto, en un promedio de 26.20, 38.41 y 51.10 respectivamente. En la escala 5 la cantidad de orificio oscila entre 50 y 60 perforaciones por fruto. A pesar del uso indiscriminado de plaguicida, no se logró un control eficaz sobre esta plaga. Es posible que por desconocimiento de la etología del insecto, o bien porque las aplicaciones de los insecticidas no fueron oportunas ni adecuadas, sin embargo, considerando las características propias de esta plaga, el control químico sigue siendo un método no muy recomendado. Por otro lado, se logró un $52.3 \%$ de frutos normales, contra un $47.7 \%$ de frutos deformes, esto implica que la población de insectos polinizadores no fue afectado a pesar del uso de estos productos, lo que demuestra una buena participación de dichos insectos. En relación al número de frutos sanos el $21.8 \%$ es muy bajo si lo comparamos con la plantación con un manejo mínimo que es del $63 \%$. Cómo se explica esto, que a pesar del empleo de plaguicida el porcentaje de frutos dañados es del $70.0 \%$. Como la reporta Pimentel y Levitan (1986) sólo una cantidad insignificante del producto químico (menos del $0.1 \%$ ) logra llegar a la plaga objetivo. La mejor recomendación sería establecer medidas preventivas y no de control.

En el monitoreo realizado en el mismo mes en la localidad de Pinoltepec, municipio de Emiliano Zapata, Ver., con un manejo mínimo de cultivo (manejo de maleza, abono orgánico y riego), se observó que la incidencia de $B$. cubensis según la escala de daño fue del $12 \%$. Pero en relación al número de frutos dañados por este insecto fue del $10 \%$. Asimismo, la incidencia del perforador del fruto o palomilla según la escala de daños muestra un $27 \%$ pero las lesiones provocadas por este insecto se consideran leves (Cuadro 2). Por el contrario, a pesar de que no se aplicó plaguicidas que en un momento dado podría haber afectado a la población de insectos polinizadores, se cosechó un $86 \%$ frutos deformes, muy significativo, comparado con la plantación bajo condiciones de manejo.

Se puede concluir que con un manejo mínimo a la que está sometida esta plantación, la incidencia de plagas y enfermedades fue menor en comparación con la plantación bajo manejo de cultivo. Lo anterior coincide con lo reportado por Cervera (2010) quién reporta que la incidencia de Bephratelloides cubensis y Cerconota anonella fue diferente en frutos de
Annona muricata procedentes de plantaciones con diferente manejo. En frutos procedentes de plantaciones con manejo emergieron en mayor proporción individuos de B. cubensis $(62.08 \%)$ en comparación con frutos sin manejo $(34.02 \%)$. Para $C$. anonella emergieron frutos procedentes de plantaciones sin manejo un $3.8 \%$ de individuos, no registrándose emergencia de individuos de esta especie en frutos procedentes de plantaciones con manejo. Diversos autores, han documentado que en el proceso de oviposición esta palomilla queda expuesta en su estadio de huevo y larva al medio ambiente hasta por 23 días lo cual los hace más susceptibles al efecto de sustancias químicas aplicadas para su control (BUSTILLO y PEÑA, 1992; MOURA y VIEIRA, 1997; PEREIRA et al., 1998). Este hecho, pudo constituir un elemento por el cual no se registró individuos en los frutos procedentes de plantaciones donde se aplican plaguicidas.

Las enfermedades al igual que las plagas que atacan a los frutales tropicales resultan de gran impacto económico debido a las pérdidas que ocasionan en el rendimiento y calidad de la cosecha. Las principales enfermedades de las anonáceas reportadas en México son: antracnosis (Colletotrichum gloeosporioides Penz), pudrición blanda, (Rhizopus stolonifer Ehr. ), Mancha negra de la hoja (Phyllosticta sp), Mancha del ápice de la hoja (Pestalotia sp.), Mancha blanca de la hoja (Macrophoma sp.), Fusarium sp y Phytopthora sp.

La enfermedad de mayor importancia en el cultivo del guanábano es la antracnosis ya que disminuye la producción y calidad de los frutos. El agente causal responsable de esta enfermedad es el hongo Colletotrichum gloeosporioides Penz, que se presenta en plántulas y plantas adultas atacando tallos, ramas, hojas, flores y frutos, ocasionando bajos rendimientos por hectárea (VIDAL, 1983 y LAVALLE, 1986).

En un estudio realizado en el estado de Tabasco, González y Castelán (2011) reportan que la severidad de la antracnosis en hojas de guanábano es variable según la época de muestreo y el agroecosistema. Los mayores porcentajes de severidad (49.0 y $11.4 \%)$ se registraron tanto en época seca como al inicio de las lluvias respectivamente. Los porcentajes de incidencia de antracnosis en frutos de guanábana que se presentaron en la época de "Nortes" variaron de 3.6 a $16.6 \%$, época que coincide con la etapa de floración y el inicio del amarre del fruto. En la época seca, el porcentaje de incidencia de antracnosis en fruto varió de 12.5 a 23\% que coincide con el desarrollo del fruto. En la época de lluvias la incidencia fue mucho mayor que en las 
dos anteriores y varió de 11.6 a 44.0\% y se presentó en frutos próximo a la cosecha.

Es importante señalar, que la antracnosis en ramas de guanábano es un problema serio en la propagación por injerto. Aquella vareta o púa infectada por este hongo difícilmente se logra el "prendimiento" del injerto. La pudrición blanda, siempre se ha reportado como agente causal al hongo del género Rhizopus stolonifer Ehr., es una enfermedad de considerable importancia, dado que ataca a frutos próximo a la cosecha y posterior a ésta, ocasionando el $50 \%$ de pérdidas más.

\section{Manejo analítico con un enfoque empírico}

\section{Bajo este modelo reduccionista las plagas y enfermedades habrá que, exterminarlas y/o combatirlas porque son las causas de los problemas.}

Comúnmente el productor acude a las tiendas de venta de productos agroquímicos y le expone al técnico (generalmente son jóvenes de reciente egreso de la Universidad, sin ninguna experiencia) su problema sobre los daños que presenta su cultivo. En base a este diagnóstico visual del productor, el técnico toma decisiones para las recomendaciones. Revisa las etiquetas de los plaguicidas para ver las instrucciones de los productos relacionadas con las plagas que controla y bajo este criterio da las recomendaciones sobre las dosis y número de aplicaciones. La verdad es que el propio productor desconoce si el daño es producto de un insecto o una enfermedad. Asimismo, no sabe diferenciar entre un insecticida y un fungicida y para qué sirven. Por otro lado, no existe un plaguicida que en su etiqueta mencione las plagas del guanábano y su control como lo hacen con otros cultivos y frutales. La realidad es que los técnicos desconocen la dinámica poblacional y su comportamiento de la plaga, el momento de la incidencia en la plantación, la severidad y época crítica de daño. Estos son indicadores que nos sirven para definir estrategia de manejo. Muchos técnicos continúan aconsejando productos organoclorados los cuales no son aconsejables principalmente por la residualidad en los productos de cosecha y otros problemas graves que han surgido de su utilización.

La falta de conocimiento por parte de los productores sobre la mejor forma de actuar contra las plagas, la época crítica de daño, las diferentes plantas hospedantes de la plaga, etc., hacen que efectúen prácticas sin su debido éxito.
En relación con la formulación de insecticidas químicos (venenos), existe poca uniformidad de criterio pues, algunos autores formulan en términos de ingrediente activo en tanto que otros utilizan formulaciones en cantidad de producto comercial; ésta desuniformidad de criterio desorienta al agricultor por falta de conocimientos que le permitan realizar las conversiones necesarias, situación que puede prestarse para formulaciones erróneas lo que causaría problemas de fitotocixidad por sobredosis o en caso contrario no lograr un efectivo control por subdosis.

Para el control de las plagas y enfermedades de las anonáceas en México, se utilizan indistintamente los siguientes productos para el problema fitosanitario. Haciendo hincapié de las recomendaciones de los técnicos con la rotación de los producto con la supuesta finalidad de no crear resistencia entre ellos.

Los insecticidas utilizados para el control de plagas en guanábano son: Malathión 1000 (malation); Horta 25 (diazinon); Dimetri 400 (dimetoato) y Siroco (cipermetrina); Hortimec 1.8\%; Matagus 34; Platino y Cyrux 20\%. El uso de plaguicida en combinación con barreras físicas y trampas ejerce buen control. En este caso se recomienda cubrir los frutos pequeños con bolsas de plástico perforada y bolsa de papel kraft impregnadas con plaguicidas (BROGLIO y BERTI, 2000). La aplicación externa a la bolsa de papel kraft de clorpirifos al $1 \%$ reduce hasta 94.5\% la infestación de frutos (HERNÁNDEZ et al., 2007). La práctica del embolsado al igual que la recolección de frutos dañados ejercen un buen control para Cerconota anonella y Bephratelloides cubensis.

Fungicidas empleados en el control de enfermedades: Atrakn (benomilo); Cupravit (oxicloruro de cobre); Cosmocel 200 (oleato cúprico); Tecto 60; Kocifol MCW; Rodeo $50 \mathrm{PH}$.

Trampeo: Las trampas McPhail (pulpa de guanábana); McPhail (jugo de guanábana); McPhail (jugo de piña) no resultaron ser efectivas en la captura del barrenador de la semilla. La captura de $B$. cubensis mediante platos de color verde con adherente sintético atrae a mayor número de adultos de esta especie (DE LOS SANTOS y DOMÍNGUEZ, 2009).

El control biológico es una herramienta que en forma natural ayuda a disminuir las poblaciones, al respecto se han encontrado algunos parasitoides y depredadores en especies de Annona en el estado de Veracruz, todo ello en vías de estudios (RUIZ, 2012 Comunicación personal ${ }^{1}$ ). 


\section{Manejo holísco con un enfoque agroecológico}

Bajo este paradigma podemos cambiar el nombre de plagas y enfermedades por indicadores de mal manejo. Los insectos, ácaros, nematodos, hongos, bacterias y virus son la consecuencia y no la causa del problema.

En el panorama científico, un nuevo paradigma ha emergido recientemente: la Teoría General de Sistemas, una teoría general para los muchos tipos de sistemas que se investigan en las ciencias naturales. Surgió en respuesta a la necesidad de hacer contrapeso a la tendencia de fragmentación y duplicación de la investigación científica. De acuerdo con Bertalanffy (1968), la teoría general de sistemas busca la integración de los diversos campos de la ciencia a través del desarrollo de principios unificadores que crecen todos los universos individuales de las ciencias.

Las frutas anonáceas actualmente debe considerarse parte constituyente de una realidad global más amplia. El campo de estas especies no es una unidad aislada. El concepto es aplicable en una fruticultura de subsistencia y a la más sofisticada y científica de ellas. Varían en estabilidad, complejidad y tamaño. Su propósito es utilizar el medio en forma sostenida para obtener frutas de consumo inmediato o transformable y estará sujeto a un diagnóstico, diseño y evaluación, que en el tiempo tenderá a modificarse. Para tener una visión holística más cercana a la realidad de un agroecosistema de las frutas anonáceas se requiere de trabajo interdisciplinario, aunado al conocimiento empírico.

En el caso del manejo integrado de plagas (MIP), éste se concibe como la selección, integración e implementación de tácticas de manejo de organismos dañinos con un enfoque de sistemas, tomando de antemano como base las consecuencias socioeconómicas y ecológicas.

Un efectivo MIP basado en esta teoría, debe ser parte integral del manejo global de un agroecosistema, de un bosque y hasta de una cuenca, y considerar la necesidad de incrementar la producción agrícola, determinar las pérdidas económicas, considerar la seguridad y riesgo a la salud humana, la calidad del ambiente, requerimientos de energéticos y el daño potencial de los organismos dañinos. Por lo tanto, los métodos de MIP pueden enmarcarse en el enfoque de sistemas. En cuanto al manejo integral de arvenses (MIA) éste debe implementarse como parte de las estrategias de manejo de plagas entomológicas $\mathrm{y}$ del agroecosistema.

La tendencia actual del manejo de los problemas entomológicos en todos los cultivos, es el manejo integrado de plagas (MIP), empleándose en forma combinada y armónica todos los recursos disponibles para reducir al máximo las poblaciones de insectos plagas abajo del nivel de daño económico.

Con el desarrollo de la Epidemiología Agrícola, se ha dado un mayor peso al análisis cuantitativo y ecológico de los diversos componentes involucrados en una epidemia y a las interacciones que se establecen entre ellos; se han desarrollados diversas técnicas y métodos cuantitativos y se han tomado prestados algunos pertenecientes a otras áreas de la ciencia como son la química, la ecología, etología, etc. Se ha pasado de un análisis específico para cada componente, a un análisis sistémico o integral y dinámico en tiempo y espacio, lo cual implica que las epidemias ocasionadas por plagas y/o enfermedades son eventos vivos, dinámicos, multivariados, en constante cambio y que sólo su estudio a través del tiempo y del espacio nos permite adquirir un conocimiento y entendimiento de su comportamiento para establecer estrategias para su manejo bajo un contexto racional y ecológico.

Respecto a la estructura de una epidemia, ésta considera al menos tres componentes interactuantes en el tiempo: patógeno, hospedante y enfermedad, que son considerados como elementos estructurales. El agroecosistema es el sistema mayor, el cual no se limita a un único cultivo o parcela, sino que espacialmente considera las parcelas de los alrededores, bosques, acahuales, etc., y temporalmente considera la secuencia de cultivos de la misma parcela. La mayoría de los investigadores en fitopatología han dedicado sus esfuerzos sólo a nivel patógeno, enfermedad y epidemia, y apenas recientemente, el sistema mayor se ha considerado como los objetivos de investigación. El enfoque de sistemas es un concepto apropiado para lograr un mejor entendimiento de las dinámicas de población de hospedantes y patógenos. La consistente aplicación del enfoque de sistemas puede también ayudar en la identificación de los posibles efectos adversos de las estrategias o tácticas de control. Los límites de análisis de un sistema dependen de los objetivos de investigación o proyecto.

En epidemiologia, el análisis de sistemas es una estrategia de investigación o marco para solucionar problemas complejos de sistemas biológicos y a sus elementos estructurales (KRANZ y HAU, 1980). Si bien hace muy poco que la ciencia de los sistemas comenzó a aplicarse al manejo de plagas, se han logrado ya algunos éxitos en la fase de aplicación práctica. El desarrollo de una buena estrategia de manejo de plagas depende de la capacidad e información que se tenga para comprender todas las interacciones significativas y 
relevantes del mundo real.

Se requiere desarrollar sistemas que aseguren que los árboles sean lo suficientemente sanos para tener ventajas sobre el aprovechamiento de agua y nutrimentos disponibles, lo cual puede conducir a una producción más sustentable. Según Cook (1986) se requiere de ocho principios para el manejo holístico de un sistema frutícola: 1) Conocer los factores limitantes de la producción del agroecosistema. 2) Mantener el contenido de materia orgánica en el suelo. 3) Usar rotación de cultivos que incluyan leguminosas forrajeras. 4) Minimizar el estrés nutricional del cultivo. 5) Usar cultivares resistentes a plagas. 6) Maximizar los beneficios de los organismos benéficos y 7) Usar plaguicidas sólo cuando sea necesario.

En la actualidad, la mayoría de los sistemas de producción no están en condiciones para un cambio radical a una fruticultura totalmente sustentable, ya que podría ocasionar un decremento en la producción de alimentos. Los cambios deben ser graduales y con el tiempo incorporar prácticas que vayan incrementando la sustentabilidad.

Los principales problemas de las anonáceas en México son: 1) No cuenta con variedades mejoradas y 2) Plagas y enfermedades. Para ello, se han iniciado algunas líneas de investigación dirigidas a contrarrestar principalmente el problema del $B$. cubensis y $C$. anonella. Se cuenta con materiales seleccionados de guanábano en donde la presencia de numerosas reminiscencia estilares en frutos pequeños favorece una protección física contra el barrenador de la semilla. Un cultivar Vidal/AE cuyo frutos presentan poca semilla y son pocos afectados por el barrenador. Existen selecciones con ciertas resistencia a la misma plaga y dentro de esta variación genética del guanábano existen tolerancia a la antracnosis y a las bajas temperaturas. Evaluación de extracto de Nim y otros. La nutrición orgánica y la incidencia de plagas y enfermedades. Evaluación de portainjertos y sus efectos en la resistencia de plagas y enfermedades. Uso de micorrizas y rizobacterias para una mayor eficiencia en la nutrición. Todos estos estudios deberán ser planeados a través de un enfoque holístico e interdisciplinario.

Cuadro 1- Características de los frutos cosechados en una plantación con manejo de cultivo

\begin{tabular}{|c|c|c|c|}
\hline Escala de daño & Prom. $\mathbf{O} / \mathbf{F}^{1}$ & Número $\mathbf{F} / \mathbf{N}^{2}$ & Número $F / \mathbf{D}^{3}$ \\
\hline 0 (Sin daño) $0 \%$ & 0 & $13(7.5 \%)$ & 25 \\
\hline $1(1-10) 12 \%$ & 6.39 & $21(12 \%)$ & 34 \\
\hline $2(11-20) 25 \%$ & 16.01 & $21(12 \%)$ & 14 \\
\hline $3(21-30) 50 \%$ & 26.20 & $9 \quad(5.2 \%)$ & 6 \\
\hline $4(31-40)(75 \%)$ & 38.41 & $10(5.7 \%)$ & 3 \\
\hline $5(41-60)(100 \%)$ & 51.10 & $17(9.8 \%)$ & 1 \\
\hline Subtotal de frutos normales & & $91(52.3 \%)$ & \\
\hline Subtotal de frutos deformes & & & $83(47.7 \%)$ \\
\hline Número de frutos sanos & $36(20.3 \%)$ & & \\
\hline Número de frutos dañados avispita & $121(70.0 \%)$ & & \\
\hline Número de frutos dañados palomilla & $17(10.0 \%)$ & & \\
\hline Total de frutos & 174 & & \\
\hline
\end{tabular}

${ }^{1}$ Promedio orificio por fruto; ${ }^{2}$ Número de frutos normales y ${ }^{3}$ Número de frutos deformes

Cuadro 2-Características de los frutos cosechados en una plantación con un manejo mínimo de cultivo.

\begin{tabular}{lc}
\hline \multicolumn{1}{c}{ Escala de daño por avispita y otras variables } & Observaciones \\
\hline $1(1-10) 12 \%$ (daños leves) & 4.89 promedio de orificios \\
Subtotal de frutos normales & $20(13 \%)$ \\
Subtotal de frutos deformes & $130(86 \%)$ \\
Número de frutos sanos & $95(63 \%)$ \\
Número de frutos dañados por avispita & $15(10 \%)$ \\
Número de frutos dañados por palomilla según escala: 2 (11-20) 25\% & $40(27 \%)$ \\
Total de frutos & 150 \\
\hline
\end{tabular}




\section{CONCLUSIONES}

1. Realmente es poca la investigación que se ha hecho en México sobre ecología, biología y etología de las plagas y enfermedades que afectan a las Anonáceas y sobre los diferentes métodos de manejo de plagas y enfermedades.

2. En México son escasas las investigaciones encaminadas al estudio sobre control biológico dirigido en las Anonáceas.

3. No existe uniformidad respecto a la formulación de insecticidas químicos y la época de aplicación, provocando la baja eficiencia de estos, debido a que su aplicación se basa al criterio del técnico o del agricultor. Situación que afecta el control eficiente de los agentes patógenos que dañan a las Anonáceas.

4. En chirimoyo y guanábano es muy poca la información bibliográfica existente sobre plagas y enfermedades, y en las demás especies de Annonas es nula la información sobre estos tópicos.

5. En México no se hacen evaluaciones de las pérdidas que ocasionan las plagas en las Anonáceas, ocasionando un desconocimiento concreto sobre los niveles de producción alcanzados por estas especies, en particular en aquellas consideradas de importancia económica para el país.

6. Se deberá implementar programas sobre el manejo integral de los agroecosistemas de las anonáceas, en principio sobre un enfoque sistémico en el patosistemas de estas especies.

7. Capacitar a profesionales en la problemática de las Anonáceas en México.

\section{REFERENCIAS}

ALOMÍA, D.G.B. Principales plagas de las anonáceas. IICA Informa, Turrialba, p.11-14, 1980.

ANDRÉS, A. J.; ANDRÉS, H.L. Biología, diversidad, conservación y uso sostenible de los recursos genéticos de annonaceae en México. Chapingo: Centro Regional Universitario Centro Occidente, Universidad Autónoma Chapingo, 2011. $152 \mathrm{p}$.
BAUTISTA, M.N. L. M. ; HERNÁNDEZ F.; LINDERAL C. Insectos de importancia agrícola poco conocidos en México. Montecillo: Instituto de Fitosanidad, Colegio de Postgraduados, 2003. 33 p.

BERTALANFFY, L. Teoría general de sistemas. México: Editorial Fondo de Cultura Económica, 1968. $311 \mathrm{p}$.

BOSCAN, D.M.M.; GODOY, F.J. Distribución geográfica de Talponia sp., Cerconota sp. y Bephratelloides sp., perforadores de flores y frutos de Guanábana en Venezuela. Agronomía Tropical, Maracay, v.39, n.6, p.319-323, 1989.

BRECHELT, A. EI manejo ecológico de plagas y enfermedades. Santiago de Chile: RAP-AL, 2004. $36 \mathrm{p}$.

BROGLIO, M.S.M.F.; BERTI, E Controle de Cerconota anonella em pomar de graviolera. Scientia Agrícola, Piracicaba, v.57, n.3, p. 557-559, 2000 .

BUSTILLO, A. E.; PEÑA, J.E. Biology and control of the Annona fruit borer Cerconota anonella Sepp (Lepidóptera:Oecophoridae). Fruits, Paris, v.47, n.1, p. $81-84,1992$.

CERVERA, R. R. A. Incidencia por estrato arbóreo de (Bephratelloides cubensis A.) y (Cerconota anonella S.) en frutos de Annona muricata L.) procedentes de plantaciones con diferente manejo en la zona centro del estado de Veracruz, México. 2010. 46 f. Tesis (Profesional) - Facultad de Ciencias Agrícolas, Campus Xalapa, Universidad Veracruzana, Vera Cruz, 2010.

COOK, R. J. Interrelationships of plant health and the sustainability of agriculture with special reference to plant disease. American Journal of Alternative Agriculture, Oxon, v.1, p. 19-24, 1986.

COTO, D. A.; SAUNDERS, J.L. Insecto plaga de la guanábana (Annona muricata). Manejo Integrado de Plagas y Agroecologia, Turrialba, v.61, p.6068, 2001.

DE LOS SANTOS M. D.; DOMÍNGUEZ, C.X. Evaluación de trampas y atrayentes para dos plagas del guanábano (Annona muricata $\mathrm{L}$.) en Blanca Espuma, La Bandera y La Esperanza, Veracruz. 2009. 65f. Tesis (Profesional) - Facultad de Ciencias Agrícolas, Campus Xalapa, Universidad Veracruzana, Vera Cruz, 2009. 
GONZÁLEZ, A. L; CASTELÁN, E. M. Incidencia de enfermedades en el cultivo de guanábana (Annona muricata L.) en Tabasco. In: ANONÁCEAS. Plantas antiguas, estudios recientes. Chiapas: Universidad de Ciencias y Artes de Chiapas, 2011. p. 413-425.

GONZÁlEZ, E. A. R; LUNA, C. L; MORENO, A. R; DE LA CRUZ, C. I.;BARAJAN, H. P. Distribución del género Annona. In: CONGRESO INTERNACIONAL DE ANONÁCEAS, 2., 1999, México. Memoria... México: Tuxtla Gutiérrez, 1999. p.85.

HERNÁNDEZ, F.L.M.; BAUTISTA, M.M.; CARRILLO, S.J.L.; SÁNCHEZ, A.H.; URÍAS, L.M.A.; SALAS, A.M.D. Control del barrenador de las semillas, Bephratelloides cubensis Ashmead (Hymenóptera-Eurytomidae) en guanábana Annona muricata L. Acta Zoológica Mexicana, Nueva Serie, México, v.24, n.001, p.199-206, 2008.

HERNÁNDEZ, F. L. M; BAUTISTA, M. M; CARRILLO, S. J. L; SÁNCHE,Z A. H; URIAS, L. M. A; SÁNCHEZ, A. H. La guanábana: plagas y su manejo. Nayarit: Fundación Produce Nayarit, 2007. 32 p. (Folleto Técnico, 1).

ILLESCA, R. C. P. Plagas en frutos del género Annona existentes en la zona centro del estado de Veracruz. 2009. 72 f. Tesis (Profesional) Facultad de Ciencias Agrícolas, Zona Xalapa, Universidad Veracruzana, Vera Cruz, 2009.

KRANZ, J.; HAU, B. Systems analysis in epidemiology. Annual Review of Phytopathology, Palo Alto, v.18, p.67-83, 1980.

LAVALLE, V. J. A. Evaluación del daño que causa las principales enfermedades en el fruto de la guanábana (Annona muricata L.) en Sihochac, Campeche. 1986. 55 f.Tesis (Profesional) - Colegio Superior de Agricultura Tropical, Tabasco. 1986.

MOURA, L. J. C.; VIEIRA, L. J. B. Manejo integrado das pragas da gravioleira. In: SÃO JOSÉ, A. R.; SOUZA, I. V. B., MORAIS, O. M.; REBOUÇAS, T. N. H (Ed.). Anonáceas: produção e mercado (pinha, graviola, atemóia e cherimóia). Bahia: Universidade Estadual do Sudoeste da Bahia, 1997. p. 214-221.
OROZCO, S. M.; LÓPEZ, P. J.; FARÍAS, L. J.; ARENAS, V. M. Sustentabilidad en el Manejo de Plagas y Enfermedades de Cultivos Agrícolas. Revista Mexicana de Fitopatología, Chapingo, v.16, n.1, p. 46-54, 1998.

PEÑA, J.E.; NAGEL, J. Effectiveness of pesticides against two tropical fruit pests. Proceedings of the Florida State Horticultural Society, v.101, p.249251, 1988..

PEREIRA, M. J. B.; DOS ANJOS, N.; EIRAS, A. E. Cortejo y cópula en la broca de la semilla de guanábana Bephratelloides pomorum (Hymenoptera: Eurytomidae). Revista de Biología Tropical, San Jose, v.46, n.1, p.105-108, 1998.

PIMENTEL, D. Amounts of pesticides reaching target pest. Journal of Agricultural and Environmental Ethics, Dordrecht, v.8, p.17-29, 1995.

PIMENTEL, D.; LEVITAN, L. Pesticides: amounts applied and amounts reaching pests. BioScience, Washington, v.36, p.86-91, 1986.

PIMENTEL, D; MCLAUGHLIN, L.; ZEPP, A.; LAKITAN, B.; KRAUS, T.; KLEINMAN, P.; VANCINI, F.; ROACH, W. J.; GRAAP, E.; KEETON, W. S.; SELIG, G. Environment and and economic effects of reducing pesticide use in agriculture. Agriculture, Ecosystems and Environment, Amsterdam, v.46, p.273-288, 1993.

PONCE, H. M.; VIDAL, H. L. Asistencia técnica sobre el cultivo de la guanábana. In: CONGRESO NACIONAL DE FRUTICULTURA, 3., 1981, Guadalajara. Memoria... 171p.

PONCE, J. M. Problemas fitosanitarios de la guanábana. In: SIMPOSIUM NACIONAL DE PARASITOLOGÍAAGRÍCOLA, 4., 1976, Veracruz. Memoria... p. 104-110.

VIDAL, H. L. Efecto de reguladores de crecimiento en la formación de frutos partenocàrpicos en la guanábana (Annona muricata L.). . In: CONGRESO NACIONAL DE FRUTICULTURA, 3., 1981, Guadalajara. Resúmenes... Guadalajara: ConafrutSerh, 1981. p. 120. 